\title{
Compound mutants for the paralogous hoxa-4, hoxb-4, and hoxd-4 genes show more complete homeotic transformations and a dose-dependent increase in the number of vertebrae transformed
}

\author{
Gerald S.B. Horan, ${ }^{1}$ Ramiro Ramírez-Solis, ${ }^{2,5}$ Mark S. Featherstone, ${ }^{3}$ Debra J. Wolgemuth, ${ }^{4}$ \\ Allan Bradley, ${ }^{2}$ and Richard R. Behringer ${ }^{1,6}$ \\ ${ }^{1}$ Department of Molecular Genetics, The University of Texas M.D. Anderson Cancer Center, Houston, Texas 77030 USA; \\ ${ }^{2}$ Department of Human and Molecular Genetics, Howard Hughes Medical Institute, Baylor College of Medicine, Houston, \\ Texas 77030 USA $_{i}{ }^{3}$ McGill Cancer Centre, Departments of Oncology and Medicine, McGill University, Montreal, Canada; \\ ${ }^{4}$ Department of Genetics and Development, The Center for Reproductive Sciences, and Department of Obstetrics and \\ Gynecology, Columbia University College of Physicians and Surgeons, New York, New York 10032 USA
}

The Hox gene products are transcription factors involved in specifying regional identity along the anteroposterior body axis. In the mouse, several single mutants for Hox genes show variably penetrant, partial homeotic transformations of vertebrae at their anterior limits of expression, suggesting that compound $H o x$ mutants might show more complete transformations with greater penetrance than the single Hox mutants. Compound mutants for the paralogous group 3 genes, hoxa-3 and hoxd-3, show deletion of a cervical vertebrae, which is not readily interpretable in terms of an alteration in regional identity. Here, we report the skeletal phenotypes of compound mutants in the group 4 Hox genes, hoxa-4, hoxb-4, and hoxd-4. Mice mutant for each of these genes were intercrossed to generate the three possible double mutant combinations and the triple mutant. In contrast to the hoxa-3, hoxd-3 double mutants, group 4 Hox compound mutants displayed clear alterations in regional identity, including a nearly complete transformation of the second cervical vertebrae toward the morphology of the first cervical vertebra in one double mutant combination. In comparing the types of homeotic transformations observed, different double mutant combinations showed different degrees of synergism. These results suggest a certain degree of functional redundancy among paralogous genes in specifying regional identity. Furthermore, there was a remarkable dose-dependent increase in the number of vertebrae transformed to a first cervical vertebra identity, including the second through the fifth cervical vertebrae in the triple mutant. Thus, these genes are required in a larger anteroposterior domain than is revealed by the single mutant phenotypes alone, such that multiple mutations in these genes result in transformations of vertebrae that are not at their anterior limit of expression.

[Key Words: Homeotic genes; skeleton; pattern formation]

Received March 2, 1995; revised version accepted June 5, 1995.

The specification of regional identity along the anteroposterior body axis of many organisms culminates in the generation of a diversity of structures along this axis. The proper specification of regional structures along this axis is dependent on the action of an evolutionarily conserved set of genes, which were first identified in Drosophila melanogaster for their ability to effect homeotic transformations, reidentification of a body segment

${ }^{5}$ Present address: Center for Extracellular Matrix Biology, Institute of Biosciences and Technology and Department of Veterinary Pathobiology, Texas A\&M University, Houston, Texas 77030 USA.

${ }^{6}$ Corresponding author.
(Lewis 1978). These homeotic genes encode putative transcription factors that share a common DNA-binding motif known as the homeo domain (Slack et al. 1993). In insects and lower chordates, these homeotic genes comprise a single cluster (which is split in Drosophila; Lewis 1978; Kaufman et al. 1980; Garcia-Fernàndez and Holland 1994|. Presumably, duplications of a single ancestral cluster (the HOM-C complex) during evolution resulted in the mouse and human genomes having four independent clusters, lettered A through D (Fig. 1A) (Scott 1993), which contain at least 38 so-called Hox genes. Thus, for a given ancestral gene in the HOM-C complex, the mouse may have up to four genes, called 
A

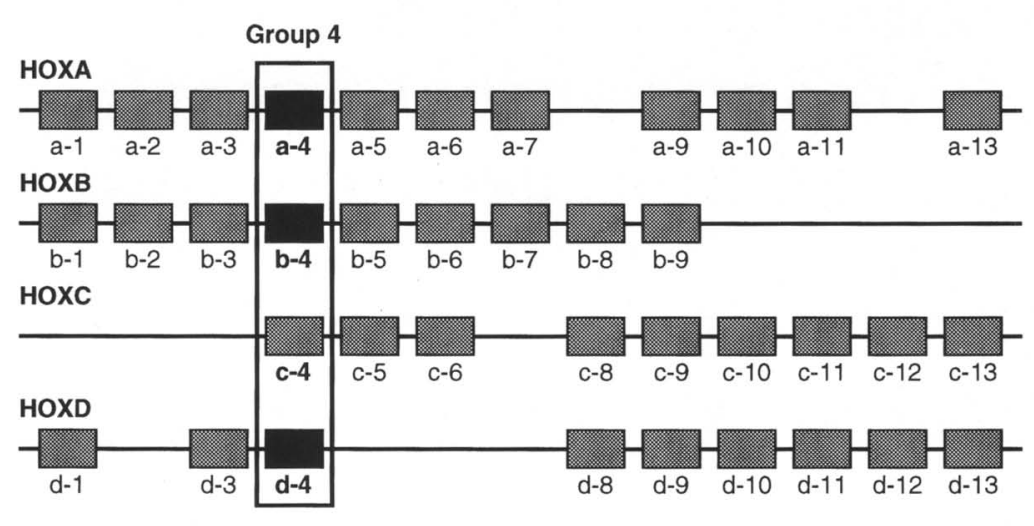

\section{$3^{\prime}$}

Anterior $\mathbf{5}^{\prime}$

Posterior
B

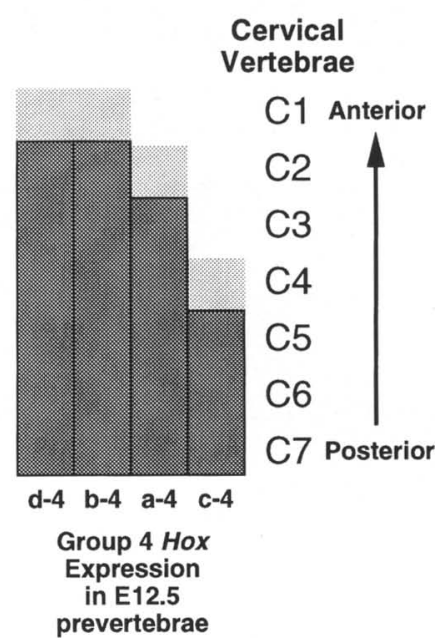

Figure 1. The Hox clusters and the group 4 Hox genes, with their anterior limits of expression in the axial skeleton. $(A)$ The $H o x$ clusters in mouse. Note that group 4 is one of only two groups with four members. (ロ) The three most anteriorly expressed members of this group that were used for combination mutant analysis. (B) Anterior limits of expression of group 4 Hox genes in E12.5 cervical prevertebrae (Gaunt et al. 1989; Geada et al. 1992), with lighter shading representing weaker expression in the anterior-most vertebrae. It should be noted that hoxb-4 expression is very weak in $\mathrm{Cl}$ and some groups have placed its anterior limit at C2 (Ramírez-Solis et al. 1993).

paralogs, which are closely related to each other and to the ancestral gene, particularly in the homeo domain.

In mouse, as in Drosophila, Hox genes are expressed along the anteroposterior axis of the embryo with discrete anterior limits that generally correlate with their relative position within a given cluster, such that the most 3 ' genes are expressed in the most anterior structures (Duboule and Dollé 1989; Graham et al. 1989). Because paralogs share analogous positions within clusters, they sometimes share similar anterior limits of expression as well. In the mouse, these discrete anterior limits are most evident in the central nervous system and in the somites and prevertebrae, which will give rise to the axial skeleton.

In Drosophila, the generation of a large number of mutant alleles of the HOM-C genes has been invaluable in elucidating the singular and combined functions of these genes (Lawrence and Morata 1994). A similar effort has been undertaken in the mouse, utilizing transgenic techniques to create both gain-of-function and loss-of-function alleles. In general, loss-of-function mutations lead to anterior transformations near the anterior limit of expression of the gene, similar to what is seen in Drosophila (Duboule and Morata 1994). Although these genes are expressed in a number of tissues in the mouse, these transformations have been primarily seen in the vertebrae of the axial skeleton. In many instances, only partial transformations of vertebral identity occur, involving the modification of some aspects of vertebral morphology whereas other aspects remain unchanged (Krumlauf 1994). In addition, these phenotypes are often incompletely penetrant, suggesting that other genes may partially compensate for their loss. The most obvious candidate genes for compensation are the paralogous genes.

There are four members of the group 4 paralogous $\operatorname{Hox}$ genes, hoxa-4, hoxb-4, hoxc-4, and hoxd-4 (Rubin et al. 1986; Wolgemuth et al. 1986; Featherstone et al. 1988; Graham et al. 1988; Geada et al. 1992). They share extensive amino acid identity in the homeo domain as well as in other domains in the protein. They have very similar expression patterns, with similar but distinct anterior limits of expression in the prevertebrae and central nervous system (Gaunt et al. 1989; Geada et al. 1992). Mice mutant for three of the group 4 genes (hoxa-4, hoxb-4, and hoxd-4) have been generated via homologous recombination in embryonic stem cells. hoxa-4 mutants show a partial transformation of the third cervical vertebra $(\mathrm{C} 3)$ to the second cervical vertebra $(\mathrm{C} 2)$ (Horan et al. 1994; Kostic and Capecchi 1994) as well as a cervical rib at C7 (Horan et al. 1994). Neonatal mice homozygous for a mutation in the first exon of hoxb-4 display partial homeotic transformations of $\mathrm{C} 2$ to $\mathrm{C} 1$, defects in the closure of the sternal rudiments, and variably penetrant neonatal lethality (Ramírez-Solis et al. 1993). Heterozygous and homozygous hoxd-4 mutants have more complete homeotic transformations of $\mathrm{C} 2$ to $\mathrm{Cl}$ than those seen in hoxb-4 mutants, as well as malformations of the neural arches of $\mathrm{Cl}$ and ectopic ossification at the basioccipital bone (Horan et al. 1995). In each of these mutants, the anterior transformations were at the respective anterior limits of expression of these genes (Fig. 1B), suggesting that they were important for establishing the regional identity of the second and third cervical vertebrae. However, each of these phenotypes 
were incompletely expressive and variably penetrant, even on an inbred genetic background (Ramírez-Solis et al. 1993; Horan et al. 1994, 1995).

To address the question of whether the incomplete penetrance and expressivity in these mutant phenotypes was the result of partial functional redundancy among the paralogs, we intercrossed these three mutants to produce the three double mutant combinations and the triple mutant. We found that in the hoxb-4, hoxd-4 double mutants, the $\mathrm{C} 2$ to $\mathrm{C} 1$ transformations became completely penetrant, with greater expressivity. Surprisingly, there was a dose-dependent increase in the number of vertebrae transformed, such that vertebrae that were not at the anterior limit of expression of these genes were also transformed. In the triple mutant hoxa$4^{-1-}$, hoxb-4 $4^{-1-}$, hoxd $-4^{-1-}$ there was a remarkably long-range transformation of identity, such that even C5 partially adopts the morphology of $\mathrm{Cl}$. Thus, these $\mathrm{Hox}$ genes are collectively required in a larger anteroposterior domain than is defined by the single mutant phenotypes. We discuss these results as they apply to current models of how Hox genes specify regional identity in the axial skeleton.

\section{Results \\ Differential viability in different double mutant combinations}

Both the hoxa-4 ${ }^{-1-}$ and hoxd $-4^{-1-}$ mice are viable $(\mathrm{Ho}$ ran et al. 1994, 1995), whereas a $50 \%$ lethality is seen in hox $b-4^{-1-}$ mice lonly hox $b-4^{r}$ mutants were used in these studies; Ramírez-Solis et al. 1993|. The viability of the three different double mutant combinations are summarized in Table 1. hoxa-4 $4^{-1-}$, hoxd $-4^{-1-}$ mice were viable, whereas hoxa-4 $4^{-1-}$, hox $b-4^{-1-}$ mice always died within the first week after birth. Although nearly all hoxb $-4^{-1-}$, hoxd $-4^{-1-}$ mice died within the first week after birth, two mice have survived for more than 6 months. The exact cause of death in the hoxa-4 $4^{-1}$, hox $b-4^{-1-}$ mutants is still being studied, but because the skeletons of some hoxa-4-1- hoxb-4 $4^{-1-}$ mice showed no major morphological abnormalities, it is likely that this lethality was because of abnormalities in tissues other than the skeleton.

hoxa-4, hoxb-4 double mutants show novel phenotypes in the cervical vertebrae

Wild-type morphology of the cervical vertebrae Compound group 4 Hox mutants on a C $57 \mathrm{BL} / 6 \times 129$ hybrid genetic background were examined for abnormalities in the axial skeleton at birth and as adults, except for hoxa$4^{-1-}$, hox $b-4^{-1-}$ mice that do not survive to adulthood. Abnormalities were limited to the cervical vertebrae. Wild-type mice have seven cervical vertebrae, designated $\mathrm{C} 1$ to $\mathrm{C} 7$ (anterior to posterior) (Fig. 2A). In general, vertebrae are composed of a vertebral body located ventral
Table 1. Genotypes of group 4 Hox mutant combinations

A. Genotypes of offspring from double heterozygote matings

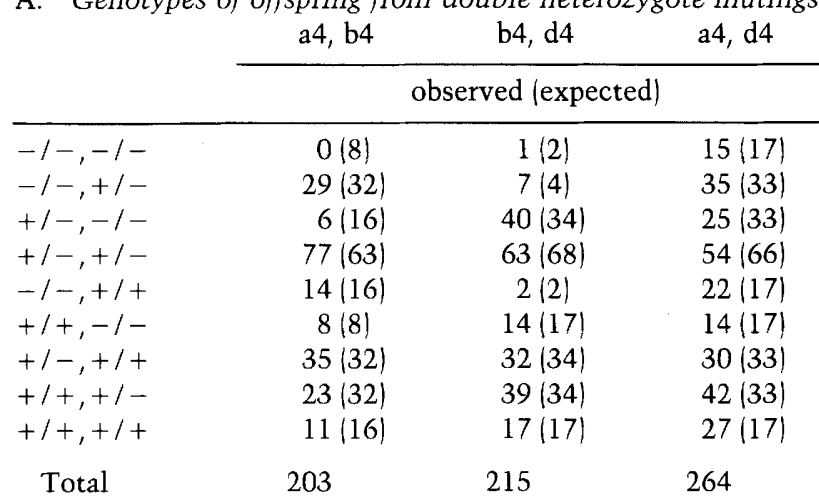

B. Genotypes of offspring from hoxb-4 heterozygote matings on $a$ hoxa-4 or hoxd-4 homozygous mutant background

\begin{tabular}{lcl} 
& $\mathrm{a} 4, \mathrm{~b} 4$ & $\mathrm{~d} 4, \mathrm{~b} 4$ \\
\hline$-1-,-1-$ & $0(12)$ & $1(7)$ \\
$-1-,+1-$ & $77(70)$ & $38(36)$ \\
$-1-,+1+$ & $40(35)$ & $22(18)$ \\
Total & 117 & 61
\end{tabular}

All of the analysis presented here involves mice on a C57BL/6 $\times 129$ hybrid genetic background, generation F3 or greater. $+1+,+1-$, and $-1-$ are wild-type, heterozygous, and homozygous mutants, respectively. (A) Observed numbers are the mice for each possible genotype surviving beyond 3 weeks of age. Expected numbers represent the predicted mendelian ratios, except for genotype combinations involving hoxb-4 homozygous mutants, which result in variably penetrant lethality. For these combinations, the number of mice homozygous for the hoxb-4 mutation and wild type for the other Hox mutations was used as an internal control to calculate the expected numbers of the other mutant combinations involving hox $b-4^{-1-}$. For this reason, the total of the expected numbers does not equal the total of the observed numbers in these combinations. (B) Because there is no such internal control in these crosses, predicted values were calculated based on the $35.7 \%$ survival of hox $b-4^{-1-}$ mice compared with wild type in the crosses of $A$.

to the neural tube and neural arches that surround the neural tube laterally and dorsally. $\mathrm{C} 1$ and $\mathrm{C} 2$, also known as the atlas and the axis, have a number of unique morphological characteristics that distinguish them from the other cervical vertebrae. First, $\mathrm{Cl}$ lacks a vertebral body (which is incorporated into the dens of C2) but possesses a ventral arch, known as the anterior arch of the atlas (aaa; Fig. 2A). In addition, $\mathrm{Cl}$ has the widest neural arches of the cervical vertebrae and a U-shaped, rostral notch in the lateral portions of the neural arches. Its foramina, through which the vertebral arteries pass, are located on the lateral surface of the vertebra and can best be seen in views of separated vertebrae (Fig. 5B, belowl. In contrast, $\mathrm{C} 2$, like the rest of the cervical vertebrae, has a vertebral body and ventral foramina but is distinct in having an anterior projection of the vertebral body known as the dens (Fig. 5B, below). The neural arches of $\mathrm{C} 2$ are slightly wider than those of $\mathrm{C} 3$ to $\mathrm{C} 7$. 


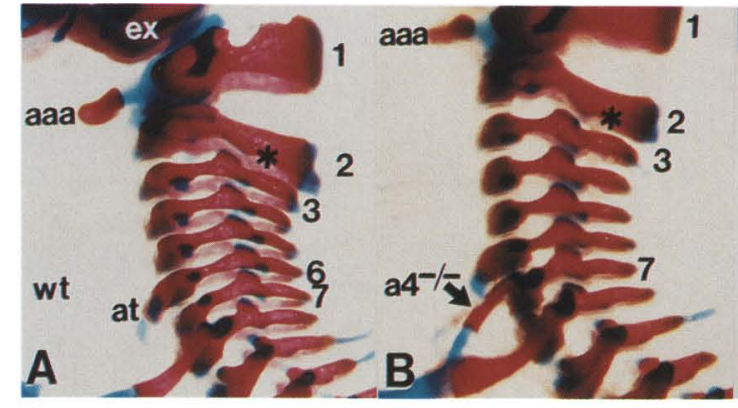

Figure 2. Skeletal abnormalities in hoxa-4, hoxb-4 and hoxa-4, hoxd-4 double mutants. In the cervical vertebrae (numbered 1 through 7) of wildtype (wt) neonates $(A)$, only $\mathrm{Cl}$ (the atlas) has an anterior arch of the atlas (aaa). C6 is morphologically distinguishable by its anterior tuberculi (at). There are articular processes on the lateral neural arches of $\mathrm{C} 2$ through $\mathrm{C} 7$. The most anterior articular process is on the caudal portion of $\mathrm{C} 2$ in the wild-type skeleton $\left({ }^{*}\right)$ and is marked by an asterisk $\left({ }^{*}\right)$ in each of the mutants $(B-F)$. In hoxa-4 mutants $(B)$, cervical ribs form on $\mathrm{C} 7$ (arrow) with low penetrance. In $\sim 20 \%$ of hoxb-4 mutants $(C)$, an aaa forms on $\mathrm{C} 2$ (arrow) and there is widening of the neural arches of $\mathrm{C} 2$. In hoxa-4 $4^{-1-}$, hoxb-4 $4^{-1}$ double mutants $(D)$, there is extensive fusion of the neural arches of $\mathrm{C} 2$ and $\mathrm{C} 3$ (short arrow). The articular processes between $\mathrm{C} 2$ and $\mathrm{C} 3$ are lost in all mice, and in $15 \%$, the rostral articular process of $\mathrm{C} 4$ is also lost (white curved arrow). In addition, the anterior tuberculi are on C7 (black curved arrow) instead of C6. The hoxd-4 phenotype $(E)$ is similar to hoxb-4 (aaa on $\mathrm{C} 2$, arrow) but involves transformation of more features of the $\mathrm{C} 2$ vertebrae and malformation of the neural arches of $\mathrm{C} 1$. In hoxa- $4^{-1-}$, hoxd-4 ${ }^{-1-}$ double mutants $(F)$, there is a low penetrance of fusion of the cartilaginous portions of the dorsal neural arches of C2 and C3 (arrow). (ex) Exoccipital bone.

C3, C4, and C5 are morphologically similar, whereas C6 is distinct in having bilateral ventral projections known as anterior tuberculi (at; Fig. 2A). C7 is morphologically similar to $\mathrm{C} 3$ through $\mathrm{C} 5$.

Novel skeletal abnormalities in hoxa-4, hoxb-4 double mutants In neonatal skeletons, hoxa-4 $4^{-1-}$ mice have a variably penetrant cervical rib on $\mathrm{C} 7$ (Fig. 2B) (Horan et al. 1994). hoxb-4 $4^{-1-}$ mice have an anterior arch of the atlas at $\mathrm{C} 2$ (Fig. $2 \mathrm{C}$ ), indicative of a partial $\mathrm{C} 2$ to $\mathrm{Cl}$ transformation (Ramírez-Solis et al. 1993). Although these phenotypes shared no common elements, the hoxa $-4^{-1-}$, hoxb $-4^{-1-}$ double mutants showed phenotypes not seen in either single mutant. In $78 \%$ of these mice, the dorsal cartilages and/or the lateral ossified portions of the neural arches of $\mathrm{C} 2$ and C3 were fused (Fig. $2 \mathrm{D}$; and Table 2A) and the lateral articular processes between $\mathrm{C} 2$ and $\mathrm{C} 3$ were lost. In some cases, the rostral articular process on $\mathrm{C} 4$ was missing as well (Fig. 2D; Table 2A).

C6 to C5 and C7 to C6 transformations in hoxa-4, hoxb-4 double mutants The formation of a cervical rib at $\mathrm{C} 7$, a phenotype seen in some hoxa-4 $4^{-1-}$ mice, was

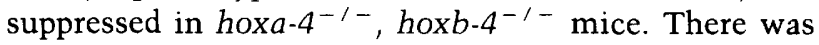
also a variably penetrant shift of the anterior tuberculi from $\mathrm{C} 6$ to $\mathrm{C} 7$ in these mice and in hoxa- $4^{+/-}$, hoxb-

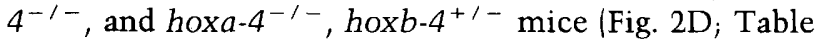
$2 \mathrm{~A}$ ), interpreted as transformations of $\mathrm{C} 7$ to $\mathrm{C} 6$ and $\mathrm{C} 6$ to $\mathrm{C} 5$. hoxa-4, hoxd-4 double mutants show a phenotype similar to hoxa-4, hoxb-4 double mutants but with lower penetrance and expressivity

Just as the hoxd-4 (Fig. 2E) mutants are similar to the hoxb-4 mutants in having an anterior arch of the atlas at C2 (Horan et al. 1995), so were hoxa-4 $4^{-1-}$, hoxd-4-1-

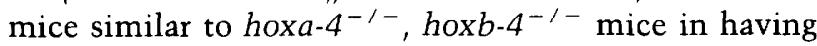
fusions of the dorsal cartilages of the neural arches of $\mathrm{C} 2$ and $\mathrm{C} 3$ (Fig. 2F). There are some $\mathrm{C} 2-\mathrm{C} 3$ fusions in hoxd-4 single mutants (Horan et al. 1995); however, those fusions resulted from the neural arches forming at abnormal angles to each other. In contrast, the fusions in the hoxa-4 $4^{-1-}$, hoxd-4 $4^{-1-}$ mice involved a piece of cartilage that extended anteroposteriorly to connect the dorsal cartilages of the neural arches that formed parallel to each other. Thus, the $\mathrm{C} 2-\mathrm{C} 3$ fusions in hoxa-4 $4^{-1}$, hoxd-4 $4^{-1-}$ mice were more similar to those of hoxa$4^{-1-}$, hoxb-4 $4^{-1-}$ mice than to those seen in hoxd-4 single mutants. However, the penetrance $(17 \%)$ of the C2-C3 neural arch fusion in hoxa-4-1-, hoxd-4 $4^{-1-}$ was significantly lower, and the lateral ossified portions of the neural arches were never fused (Fig. 2F; Table 2B) as they were in many of the hoxa-4 $4^{-1-}$, hox $b-4^{-1-}$ mice. There was also no loss of the rostral articular process of C4 (Fig. 2F; Table 2B). Furthermore, the cervical rib phenotype was not suppressed, and the $\mathrm{C} 7$ to $\mathrm{C} 6$ and $\mathrm{C} 6$ to C5 transformations were not seen. In most of the hoxa$4^{-1-}$, hox $d-4^{-1-}$ skeletons, the phenotype was simply an addition of the two single mutant phenotypes. Taken together, these results seem to indicate that there was 
Table 2. Percent penetrance of neonatal skeletal abnormalities in group 4 Hox double mutants

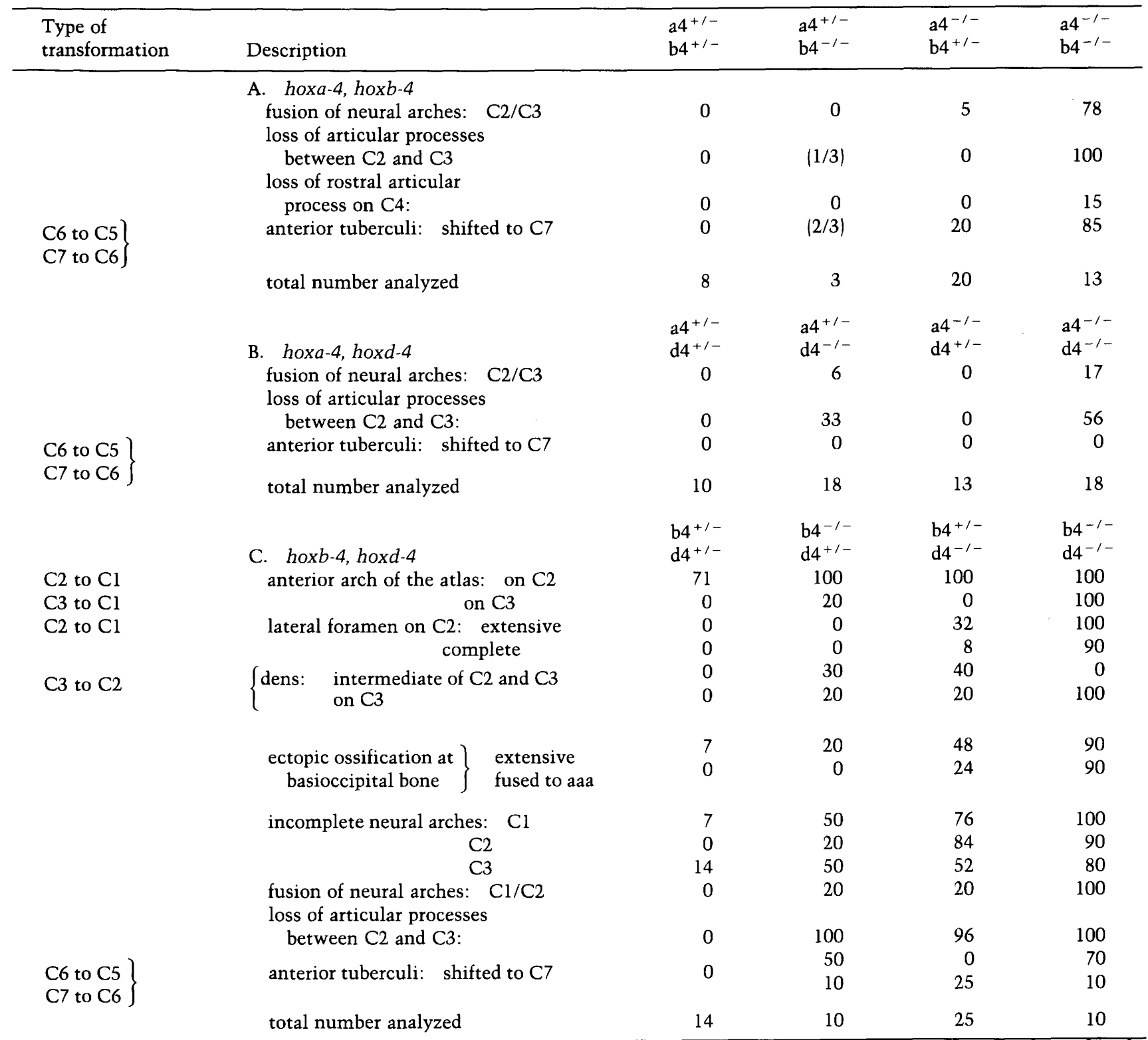

All abnormalities listed are either novel to the double mutants, or the penetrance of the abnormalities is significantly changed in the double mutants when compared with the single mutants. (A) Abnormalities in hoxa-4 $4^{+1-}$, hox $b-4^{-1-}$ mice are expressed as a fraction rather than as a percentage penetrance because of the low number of animals examined.

less synergism in the hoxa-4, hoxd-4 combination than in the hoxa-4, hoxb-4 combination.

In adult skeletons, the C3 to C2 transformation shows hoxa-4 dosage dependence on a hoxb-4 $4^{-1-}$ or hoxd-4 ${ }^{-1}$ - background

Because hoxa-4-1- mice exhibit a partial C3 to C2 transformation that is only evident in adult skeletons (Horan et al. 1994; Kostic and Capecchi 1994), mutant skeletons were also analyzed at adulthood. Wild-type and hoxa $-4^{+1-}$ mice have large processus spinosi on the dorsal surface of $\mathrm{C} 2$ but never on C3 (Fig. 3A). hoxa-
$4^{-1-}$ mice (Fig. 3B) have large processus spinosi on both $\mathrm{C} 2$ and $\mathrm{C} 3$. hoxa-4 $4^{+1-}$, hox $b-4^{-1-}$ mice also had processus spinosi on both $\mathrm{C} 2$ and $\mathrm{C} 3$ (Fig. 3C). Similarly, this $\mathrm{C} 3$ to $\mathrm{C} 2$ transformation was seen in hoxa. $4^{+1-}$, hoxd-4 $4^{-1}$ mice (Fig. 3D). Thus, this phenotype revealed a dose-dependent requirement for hoxa-4 on a hoxb$4^{-1-}$ or a hoxd-4 $4^{-1-}$ background.

More complete and penetrant homeotic transformations in hoxb-4, hoxd-4 double mutants are also dose-dependent

C2 to C1 transformations in hoxb-4, hoxd-4 mutants In single mutants for both hoxb-4 and hoxd-4, 


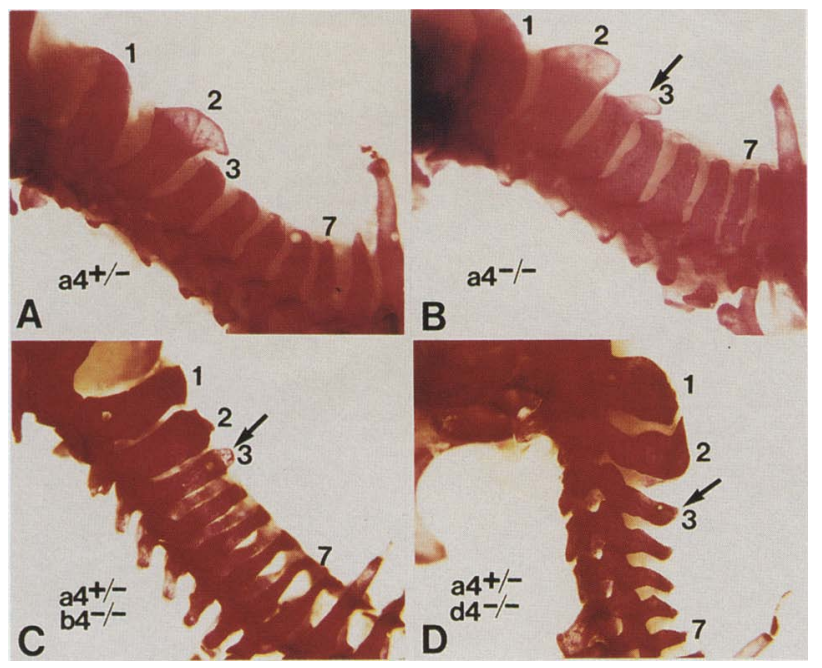

Figure 3. Dose-dependent requirement for hoxa-4 on a hoxb-4 or hoxd-4 mutant background. In adult skeletons, there is a large processus spinosus on $\mathrm{C} 2$ but never on $\mathrm{C} 3$ in wild-type and hoxa- $4^{+1-}$ mice $(A)$. In hoxa-4 $4^{-1-}$ mice $(B)$, a processus spinosus forms on C3 (arrow), a partial transformation of C3 to C2. Processus spinosi are not present on $\mathrm{C} 3$ of hox $b-4^{-1-}$ mice but are present (arrow) in hoxa- $4^{+1-}$, hoxb-4 $4^{-1-}$ mice $(C)$. A processus spinosus also forms on C3 (arrow) of hoxa-4 $4^{+1}$, hoxd$4^{-1-}$ mice $(D)$. The orientation of the cervical vertebrae in $D$ is slightly different from $A-C$ to more easily see the processus spinosus on $\mathrm{C} 3$.

there are partial transformations of $\mathrm{C} 2$ to $\mathrm{C} 1$. In neonatal skeletons, $\sim 20 \%$ and $33 \%$ of hoxb-4 and hoxd -4 single mutant mice, respectively, have an anterior arch of the atlas and widened neural arches on C2 (Ramírez-Solis et al. 1993; Horan et al. 1995). In all of the hoxb-4, hoxd-4 double mutant mice (Fig. 4C; Table 2C), C2 was almost completely transformed to a $\mathrm{Cl}$ identity, as defined by five distinct morphological criteria. The $\mathrm{C} 2$ of double mutants had an anterior arch of the atlas, widened neural arches, complete lateral foramina, loss of the vertebral body, and loss of the caudal articular process (Fig. 5B;

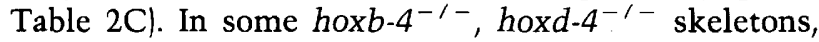
the neural arches of $\mathrm{C} 2$ were malformed. However, in other skeletons, distinctive U-shaped rostral notches, normally seen only on $\mathrm{Cl}$, were seen in the neural arches of $\mathrm{C} 2$, such that the only element missing from a complete transformation was the lack of fusion at the dorsal cartilages of the neural arches. In addition, the neural arches of $\mathrm{Cl}$ and $\mathrm{C} 2$ were often fused, similar to the

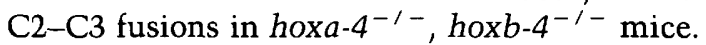

C3 to C1 and C3 to C2 transformations in hoxb-4, hoxd-4 mutants Unexpectedly, an anterior arch of the atlas also formed on $\mathrm{C} 3$, indicative of a $\mathrm{C} 3$ to $\mathrm{Cl}$ transformation. The vertebral body of $\mathrm{C} 2$ was incorporated into an extended dens on $\mathrm{C} 3$ (Fig. 5B; Table 2C), which was interpreted as a $\mathrm{C} 3$ to $\mathrm{C} 2$ transformation.

Dose-dependent intermediates in hoxb-4, hoxd-4 mutants The hoxb-4 $4^{+1-}$, hoxd-4 $-4^{-1-}$ (Fig. 4A) and hoxb$4^{-1-}$, hoxd $-4^{+1-}$ mice (Fig. 4B) represented an intermediate state between the single and double mutants, in terms of both the penetrance and expressivity of these transformations (Table 2C). The anterior arch of the atlas at $\mathrm{C} 2$ phenotype was completely penetrant in these mice, and other aspects of the $\mathrm{C} 2$ to $\mathrm{Cl}$ transformation were more penetrant (Table $2 \mathrm{C}$ ). Thus, as the number of wild-type alleles of hoxb-4 and hoxd-4 was incrementally decreased, there was a corresponding incremental shift in the position at which C1- and C2-specific structures are formed. For instance, the dens always originates from $\mathrm{C} 2$ in wild-type mice (Fig. 5B). It sometimes
Figure 4. Stepwise anterior transformations in hoxb-4, hoxd-4 double mutants and hoxa-4, hoxb-4, hoxd-4 triple mutants. An anterior arch of the atlas (aaa), a $\mathrm{Cl}$ type structure, forms on progressively more posterior vertebrae (straight arrows) as more group 4 Hox mutations are combined, going from hoxb- $4^{+1-}$, hoxd $-4^{-1-}$ (A) to hoxb-4 $4^{-1-}$, hoxd-4 $4^{+1-}(B)$ to hoxb$4^{-1-}$, hoxd $-4^{-1-}(C)$ to hoxa-4 $4^{+1-}$, hoxb $4^{-1-}$, hoxd-4 $4^{-1-}(D)$ to hoxa-4 $4^{-1-}$, hoxb$4^{-1-}$, hoxd-4 $4^{-1-}(E)$. Similarly, the position of the most anterior articular process $(*)$ is shifted to more posterior vertebrae in $(A-E)$. In addition, in hoxb-4 $4^{-1-}$, hoxd$4^{-1-}$ double mutant mice $(C)$, the neural arches of $\mathrm{Cl}$ are always incomplete and often severely shortened (open arrow). There can also be fusion of the neural arches of $\mathrm{C} 1$ and $\mathrm{C} 2$ and shifting of the anterior tuberculi to $C 7$ (curved arrow). (ex) Exoccipital bone.

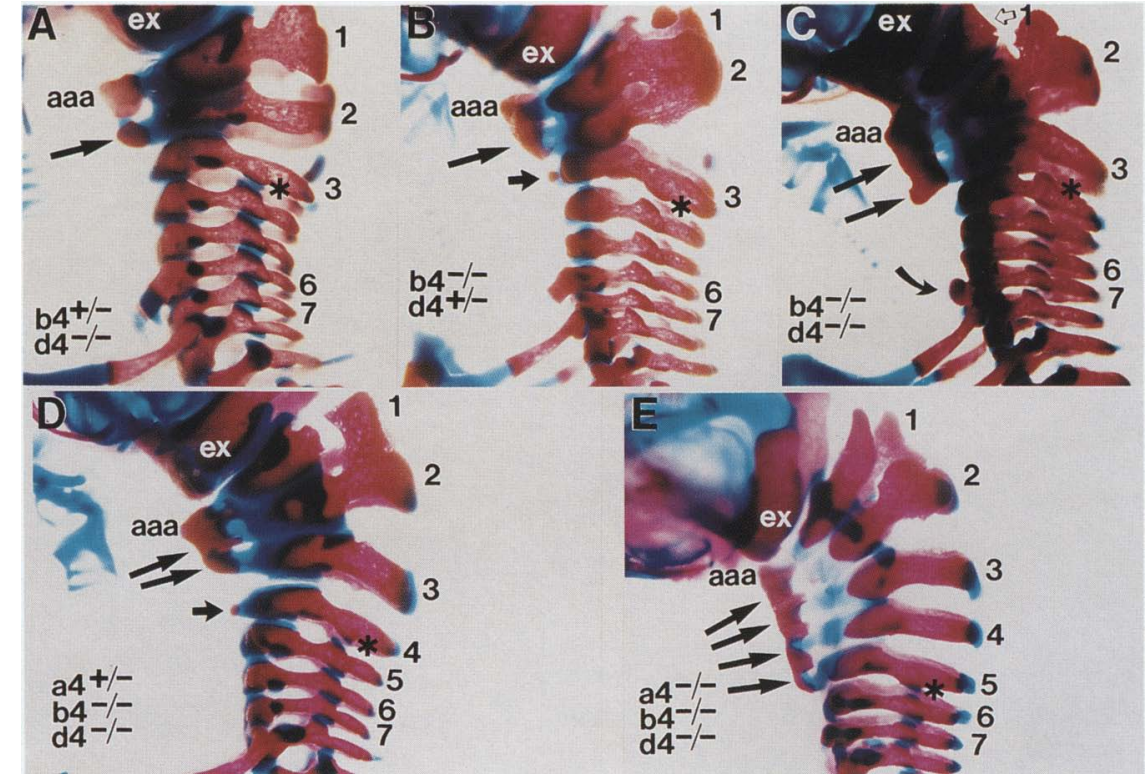




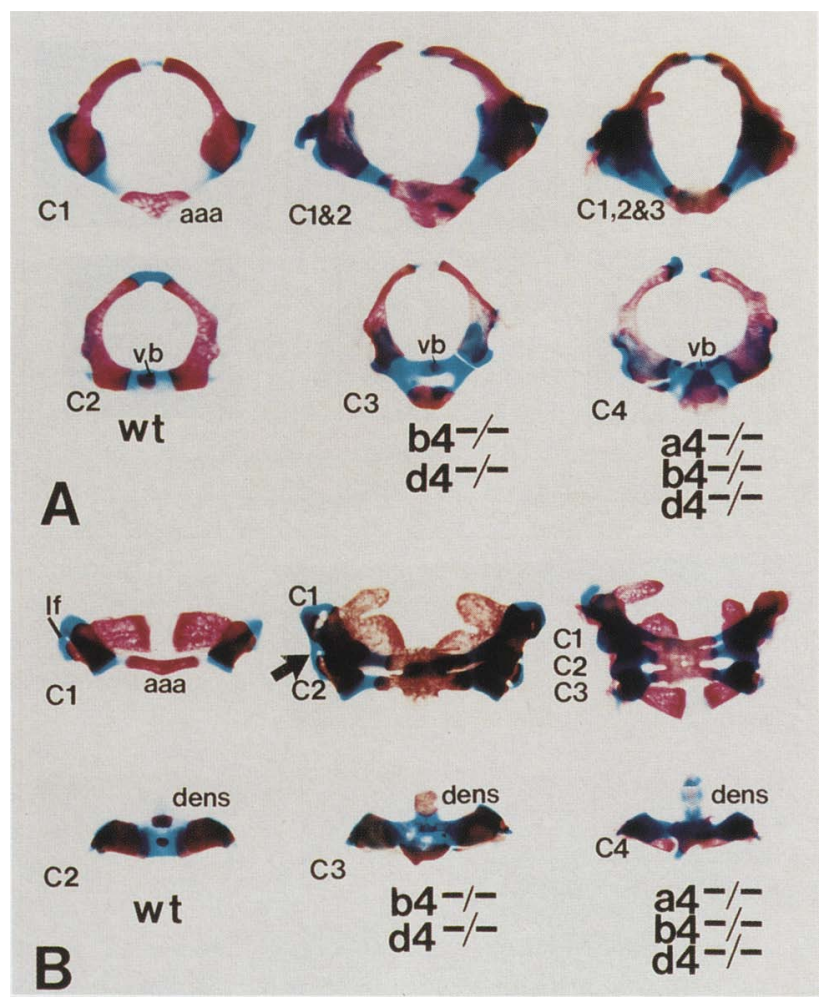

Figure 5. $\mathrm{Cl}$ - and $\mathrm{C} 2$-specific structures form on progressively more posterior vertebrae in hoxb-4, hoxd -4 double mutants and hoxa-4, hox $b-4$, hoxd -4 triple mutants. In a rostral view of partially separated vertebrae $(A)$, the vertebral body (vb) of a wildtype (wt) C2 can clearly be seen. The shape of both $\mathrm{C} 1$ and $\mathrm{C} 2$ (fused) of hoxb-4 $4^{-1-}$, hoxd $-4^{-1-}$ double mutant mice is similar to a wild-type $\mathrm{Cl}$, including absence of a vertebral body. In hoxa- $4^{-1-}$, hoxb-4 $4^{-1-}$, hoxd-4 $4^{-1-}$ triple mutant mice, C1, C2, and $\mathrm{C} 3$ (fused) all lack vertebral bodies. In a ventral view $(B)$, the dens, a structure that projects anteriorly from the vertebral body of $\mathrm{C} 2$, is shifted to $\mathrm{C} 3$ in hoxb-4 $4^{-1-}$, hoxd-4 $4^{-1-}$ mice and to C4 in hoxa-4 $4^{-1-}$, hoxb $-4^{-1-}$, hoxd-4 $4^{-1-}$ mice (some mechanical breakage of the dens occurred during separation of vertebrae). The dens at $\mathrm{C} 3$ or $\mathrm{C} 4$ is more extensive than a dens at $\mathrm{C} 2$, presumably because the vertebral bodies of $\mathrm{C} 2$ and $\mathrm{C} 3$ have been incorporated into this structure. Lateral foramina (lf) form on $\mathrm{C} 1$ in wild-type mice, on $\mathrm{C} 2$ (arrow) in hoxb-4 $4^{-1-}$, hoxd$4^{-1-}$ double mutant mice, and on C2, C3 and C4 of hoxa-4 $4^{-1-}$, hoxb-4 $4^{-1-}$, hoxd $-4^{-1-}$ triple mutant mice (not visible in this view).

originated from $\mathrm{C} 2$ on one side and from $\mathrm{C} 3$ on the other

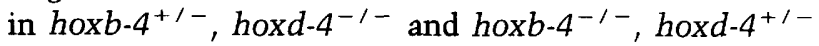
mice, and it always originated from $\mathrm{C} 3$ in hoxb-4 $4^{-1-}$, hoxd-4 ${ }^{-1-}$ mice (Fig. 5B; Table 2C).

C6 to C5 and C7 to C6 transformations in hoxb-4, hoxd-4 mutants In addition to transformations toward a $\mathrm{Cl}$ identity, hoxb-4, hoxd-4 mice also showed transformations of $\mathrm{C} 6$ to $\mathrm{C} 5$ and $\mathrm{C} 7$ to $\mathrm{C} 6$, as seen in hoxa4, hoxb-4 mice. This was seen as a shift in the position of the anterior tuberculi from $\mathrm{C} 6$ to $\mathrm{C} 7$ (Fig. $4 \mathrm{C}$; Table $2 \mathrm{C}$ ).

Other skeletal abnormalities in hoxb-4, hoxd-4 mutants Another abnormality that is specific to hoxd-4 mutant mice in the single mutants was exacerbated in the hoxb-4, hoxd-4 double mutants. Ectopic ossification at the basioccipital bone, which was minimal in hoxd$4^{-1-}$ mice, was more extensive in the hoxb-4 $4^{-1-}$, hoxd $4^{-1-}$ mice, such that a bony bridge often fuses the basioccipital bone to the anterior arch of the atlas (Table 2C).

As was mentioned previously, the $\mathrm{C} 2$ neural arches of hoxb $-4^{-1-}$, hoxd $-4^{-1-}$ mice often failed to fuse at the dorsal cartilages. These incomplete neural arches were also seen on $\mathrm{Cl}$ and C3 (Table 2C). This phenotype was most severe in the $\mathrm{Cl}$ neural arches of hoxb-4-1-, hoxd$4^{-1-}$ mice, which were often severely reduced not just dorsally but laterally as well (Fig. $4 C_{i}$ Table $2 \mathrm{C}$ ).

hoxa-4, hoxb-4, hoxd-4 triple mutants show a greatly expanded domain of anterior transformations toward a C1 identity

The number of vertebrae transformed to a $\mathrm{Cl}$ identity increased remarkably in the triple mutants. Specifically,

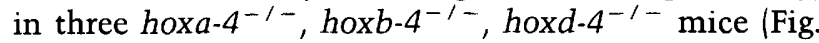
$4 \mathrm{E})$, there were anterior arches of the atlas on $\mathrm{Cl}$ through $\mathrm{C} 5$ and lateral foramina on $\mathrm{C} 2, \mathrm{C} 3$, and C4. The vertebral bodies of both $\mathrm{C} 2$ and $\mathrm{C} 3$ were incorporated into an extended dens on $\mathrm{C} 4$ (Fig. 5A,B). Therefore, in the absence of wild-type alleles of three of the group 4 Hox genes, the five most anterior cervical vertebrae assumed a nearly complete or partial $\mathrm{Cl}$ identity. In addition, small anterior arches of the atlas were present on $\mathrm{C} 4$ in 4 of 6 hoxa- $4^{+/-}$, hoxb $-4^{-1-}$, hoxd-4 $4^{-1-}$ (Fig. 4D) mice. Hence, even though the hoxa-4 mutation never produced transformations to a C1-type vertebral element (anterior arch of the atlas or lateral foramen) in either of the double mutant combinations, these transformations were seen for the first time at $\mathrm{C} 4$ and $\mathrm{C} 5$ when the hoxa-4 mutation was combined with the hoxb-4 and hoxd-4 mutations.

C6 to $C 5$ and $C 7$ to $C 6$ transformations in hoxa-4, hoxb4, hoxd-4 mutants In hoxa-4-1-, hoxb-4-1-, hoxd$4^{-1-}$ mice, the anterior tuberculi were always absent from C6, interpreted as a C6 to C5 transformation. In two of three mice, the anterior tuberculi were very small on one side of $\mathrm{C} 7$, indicative of a transformation of $\mathrm{C7}$ to C6, whereas in the third mouse, they were completely absent from $\mathrm{C} 7$. This absence of the anterior tuberculi from $\mathrm{C} 7$ may represent either a lack of transformation at $\mathrm{C} 7$ or a transformation of $\mathrm{C} 7$ to $\mathrm{C} 5$.

hoxb-5 expression is unchanged in hoxa-4, hoxb-4 and hoxb-4, hoxd-4 double mutants

The $\mathrm{C} 6$ to $\mathrm{C} 5$ and $\mathrm{C} 7$ to $\mathrm{C} 6$ transformations in hoxa-4

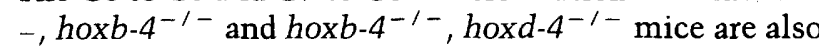
found in hoxb-5 ${ }^{-1-}$ mice (Rancourt et al. 1995). Thus, these phenotypes could be the result of altered hoxb-5 expression in these group 4 double mutants. Therefore, we immunostained hoxa-4 $4^{-1-}$, hoxb-4 $4^{-1-}$ and hoxb- 
$4^{-1-}$, hoxd $-4^{-1-}$ embryos at embryonic day 12.5 (E12.5) with a hoxb-5 antibody (Wall et al. 1992) to examine the distribution of hoxb-5 protein. In all of the embryos examined, the spatial distribution of hoxb-5 protein in the double mutants was similar to that observed in the control embryos (Fig. 6; data not shown) and appeared to be unchanged in the affected prevertebrae, pv 6 and pv 7 (Wall et al. 1992; Rancourt et al. 1995).

\section{Discussion}

Dosage-dependent increases in the expressivity and penetrance of homeotic transformations in compound mutants suggest functional redundancy among paralogs in specifying regional identity

In the other compound Hox mutants reported, the link between mutant phenotypes and changes in regional identity is less clear. In hoxa-3, hoxd-3 double mutants, the deletion of $\mathrm{Cl}$ structures seen in hoxd $-3^{-1-}$ mutants is exacerbated such that there is a nearly complete deletion of $\mathrm{Cl}$ in the double mutant; however, there is no clear homeotic transformation (Condie and Capecchi 1994). In hoxb-5/hoxb-6 transheterozygotes, there is ho-

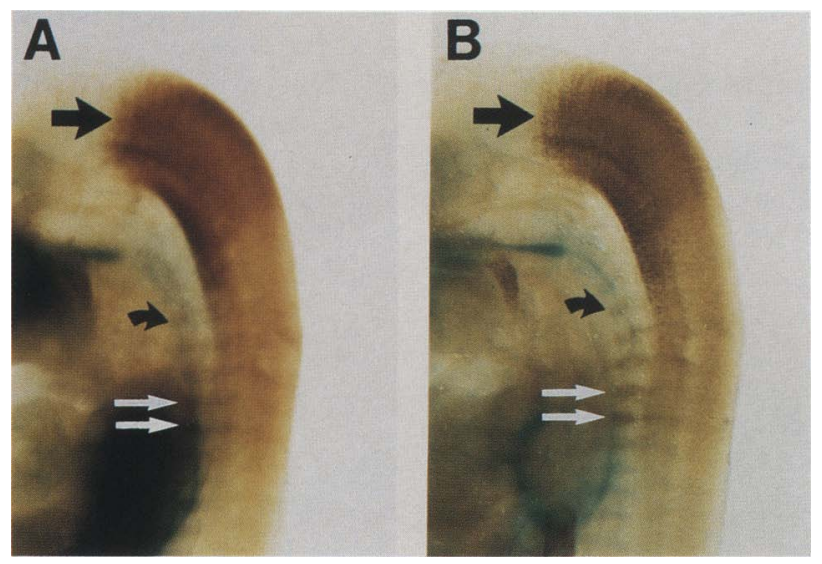

Figure 6. Expression of hoxb-5 in E12.5 control and hoxa-4, hox $b .4$ double mutant embryos. Lateral views of hemisected hoxa-4 single mutant $(A)$ and hoxa -4 , hoxb-4 double mutant $(B)$ embryos immunostained for hoxb-5 protein and counterstained with alcian blue to visualize the cartilaginous skeleton. Although the strength of the antibody staining among different embryos of all genotypes was variable, no differences in the spatial distribution of hoxb-5 protein were observed in the prevertebrae of the double mutants. Staining was weak in prevertebra 2 (pv 2) stronger in pv 3 (curved arrow) through pv 7, and weak in pv 8 . The prevertebrae that show a similar phenotype in hoxb-5 mutants and in these double mutants are pv 6 and pv 7 (white arrows). However, no differences in hoxb-5 antibody staining were detected in these prevertebrae. Similarly, no differences were found in hox $b-4$, hoxd -4 double mutant embryos (data not shown). The hoxb-5 protein localization in these embryos was identical to that observed in wild-type embryos (Wall et al. 1992; Rancourt et al. 1995). The anterior limits of hoxb-5 expression in the hindbrain of controls and the double mutants were identical (large black arrow). meotic transformation similar to that seen in hox $b-5^{-1-}$ and hoxb-6 $6^{-1-}$ mutants (Rancourt et al. 1995). Neither case, though, revealed a requirement for the genes in regions not affected in either of the single mutants, nor did they result in more complete homeotic transformation. In contrast, the homeotic transformations seen in compound mutants of the group 4 paralogous Hox genes can be readily interpreted as more complete transformations of vertebral morphology, consistent with more complete changes in regional identity. Furthermore, the homeotic transformations seen in each double mutant combination and in the triple mutant were clearly dosage dependent. Given that these genes encode similar proteins with similar expression patterns, this dosage dependence probably reflects a certain degree of functional redundancy in terms of transcriptional regulation of the same downstream target genes. However, the possibility that these genes act through parallel pathways cannot be excluded.

\section{Compound mutant phenotypes reveal a larger functional domain than is seen in the single mutant phenotypes}

In addition to an increase in the expressivity and penetrance of homeotic transformations, the number of vertebrae transformed was also increased in combination mutants. This suggests that the domain of activity for the group 4 genes extends beyond that which is defined by the single mutant phenotypes. This was most dramatically visualized by the formation of an anterior arch of the atlas, a Cl-specific structure, in the more posterior cervical vertebrae (Fig. 7). This Cl-type transformation occurred at more posterior vertebrae in a dose-dependent fashion as the hoxb-4 and hoxd-4 mutations were combined and as the hoxa-4 mutation was added to the hoxb4, hoxd-4 mutant background, such that only $\mathrm{C} 2$ was transformed in the single mutant, both C2 and C3 were transformed in the double mutant, and C2, C3, C4, and C5 were transformed in the triple mutant.

This expansion in the affected domain was also evident in the most posterior cervical vertebrae, C6 and C7. As mentioned previously, both the hoxb-4, hoxd -4 and the hoxa-4, hoxb-4 double mutants showed transformations at $\mathrm{C} 6$ and $\mathrm{C} 7$ similar to those seen in hoxb-5 single mutants (Rancourt et al. 1995). In addition, hoxa-5 mutants showed the $\mathrm{C} 6$ to $\mathrm{C} 5$ transformations but not the C7 to C6 transformations (Jeanotte et al. 1993), and hoxb-6 mutants showed a low penetrance of both types of transformations, but never bilaterally (Rancourt et al. 1995). Thus, whereas the formation of anterior arches of the atlas at more posterior vertebrae was unique to group 4 Hox mutants, certain double mutant combinations produced phenotypes in the posterior region of their functional domain that were similar to the phenotypes of single mutants for more posterior Hox genes.

A similar interpretation applies to the shortening of the neural arches of $\mathrm{C} 1, \mathrm{C} 2$, and $\mathrm{C} 3$ in the hoxb-4, hoxd-4 double mutants. The sometimes severely shortened neural arches of $\mathrm{C} 1$ resembled the most severely 


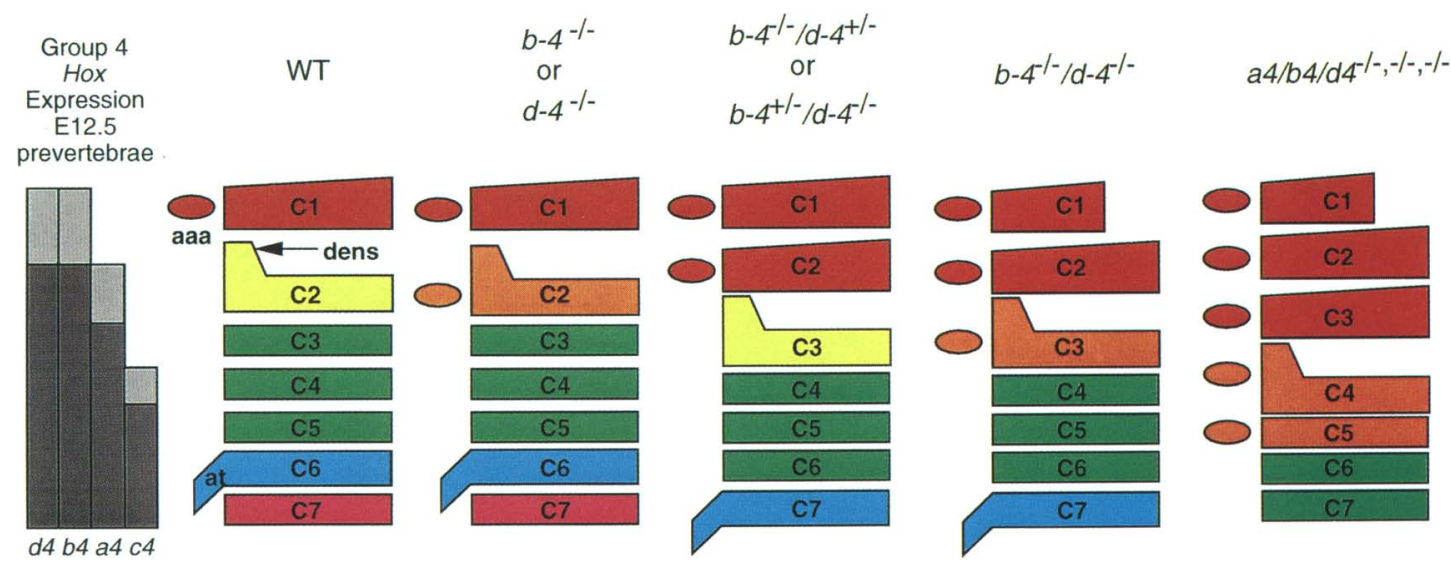

Figure 7. Schematic representation of the progressive anterior transformations of cervical vertebrae in group 4 Hox mutants. The anterior limits of group 4 Hox gene expression in E12.5 prevertebrae (pv) is depicted at left, with lighter shading representing weaker expression in the anteriormost vertebrae. Moving from left to right, the number of group 4 wild-type alleles decreases, whereas the extent of anteriorization of the cervical vertebrae increases. The transformation to a $\mathrm{Cl}$ identity is nearly complete in those prevertebrae for which group 4 Hox expression is completely lost. For example, in hoxa-4, hoxb-4, hoxd-4 triple mutants, there are no wild-type group 4 Hox genes expressed in $\mathrm{C} 2$ and $\mathrm{C} 3$, and these vertebrae are completely transformed to a $\mathrm{Cl}$ identity. hoxc-4 and the group 5 Hox genes are expressed in $\mathrm{C} 4$ and $\mathrm{C} 5$, which show partial transformations to a $\mathrm{Cl}$ identity. The group 5 genes are more strongly expressed in $\mathrm{C} 6$ and $\mathrm{C} 7$, which are never transformed to a $\mathrm{Cl}$ identity; however, their morphological changes are consistent with anterior transformations.

affected hoxd-3 single mutants /Condie and Capecchi 1993). Thus, hoxb-4, hoxd-4 double mutants showed a phenotype in the most anterior region of their functional domain that was similar to the phenotype of the single mutant for a more anterior Hox gene.

A final example of overlapping phenotypes was the fusion of the neural arches of $\mathrm{C} 1$ and $\mathrm{C} 2$ in hoxb-4, hoxd-4 and of C2 and C3 in hoxa-4, hoxb-4 mutants. These fusions were similar to the fusion of $\mathrm{Cl}$ and the exoccipital bone in hoxd-3 mutants (Condie and Capecchi 1993). These mutant combinations therefore produced similar phenotypes in different anteroposterior regions that correspond with the different anterior limits of expression of the genes.

One possible explanation for these overlapping phenotypes is that the expression of these other Hox genes is altered in the double mutants. This could be because group 4 Hox genes are directly required for proper regulation of the expression of these other Hox genes, or it could be the result of more indirect mechanisms. The hoxa-4, hoxb-4 double mutants, the hoxb-4, hoxd-4 double mutants, and the hoxb-5 single mutants (Rancourt et al. 1995) showed the most extensive similarities of any of these overlapping phenotypes, and thus, hoxb-5 represented the best candidate for having altered expression in the double mutants. However, whole-mount immunostaining of E12.5 embryos revealed no changes in the spatial distribution of hoxb-5 protein in both double mutant combinations, notably in the affected prevertebrae, pv 6 and pv 7. Thus, these transformations are not the result of a complete loss of hoxb-5 expression throughout development. We cannot, however, rule out the possibility that subtle quantitative changes in the expression of hoxb-5 or other Hox genes at different stages of development play a role in effecting these types of transformations.

\section{Different double mutant combinations show different degrees of synergism}

Although the different group 4 Hox combination mutants show extensive similarities with each other and with neighboring nonparalogous Hox genes, this study also permits us to analyze the differences between the three different mutant combinations. For instance, although hoxb-4 and hoxd-4 have almost identical anterior limits of expression and overlapping single mutant phenotypes, their double mutant combinations with hoxa-4 showed distinct phenotypes. Specifically, the hoxa-4, hoxb-4 skeletal phenotype seemed to show greater synergism than did the hoxa-4, hoxd-4 skeletal phenotype. These differences could be the result of small differences between hoxb-4 and hoxd-4 in their timing, levels, and anterior limits of expression or functional differences in the proteins. It is interesting to note, however, that in comparing the single mutant phenotypes of these three genes, hoxd-4 affects the most anterior structures, whereas hoxa-4 affects the most posterior structures. Thus, the least synergism was seen in the double mutant combination for which the affected domains of the single mutants are most separated.

\section{Paralogous Hox genes and axial patterning}

These novel observations suggest certain refinements to the current hypotheses of how Hox genes cooperatively pattern the axial skeleton. For example, based on the loss of the dens at $\mathrm{C} 2$ in hoxd-3 mutants and the formation of 
the anterior arch of the atlas in hoxb-4 mutants, it was suggested that Hox genes might cooperatively pattern vertebrae by separately specifying different morphologic features of the same vertebrae (Krumlauf 1994). Although this may still be one way in which these genes cooperatively specify identity, the hoxb-4, hoxd-4 mutants show more complete transformations of all aspects of vertebral morphology in double mutants, even for structures that are not affected in the hoxb-4 mutant (e.g., the lateral foramina) and for structures not affected in either single mutant (e.g., the incorporation of the vertebral body of $\mathrm{C} 2$ into a dens on C3). This suggests that there is not such a fine division of labor in specifying individual structures but rather a more extensive cooperativity in which several Hox genes with similar anterior limits of expression may each play a role in the proper specification of many aspects of vertebral morphology, whether directly or indirectly. Likewise, the similarities between the group 4 compound mutant phenotypes and the mutant phenotypes of neighboring nonparalogous genes suggest that this cooperativity exists among nonparalogous genes as well.

The posterior prevalence model has been useful in describing the action of Hox genes in vertebrates (Duboule 1991). In this model, genes with more posterior limits of expression are dominant in specifying identity over more anterior genes expressed in the same region. Thus, it suggests a functional hierarchy among vertebrate $H O X$ genes similar to that seen in Drosophila. This model predicts that loss-of-function mutations should produce transformations only at their anterior limit of expression and not in more posterior vertebrae where more posterior Hox genes are expressed. The complete anterior transformations of $\mathrm{C} 2$ and $\mathrm{C} 3$, which are at the anterior limit of hoxa-4, hoxb-4, and hoxd-4 expression, and the lack of transformations in the thoracic vertebrae of group 4 compound mutants are in agreement with this model. However, the transformations of $\mathrm{C} 5, \mathrm{C} 6$, and $\mathrm{C} 7$, where more posterior Hox genes are strongly expressed (Gaunt et al. 1988, 1990; Graham et al. 1989), are apparently inconsistent with this model. It has been proposed, though, that there is a quantitative aspect to this model, in which relative amounts of Hox gene products may be important in determining this functional hierarchy, perhaps by direct competition among Hox genes (Duboule and Morata 1994). If we interpret our mutant phenotypes using this more quantitative approach, then we would expect that combining mutations in Hox genes with very similar anterior limits of expression should produce a stronger anteriorizing effect, owing to decreased competition with more anterior genes. This effect would then be moderated in progressively more posterior vertebrae where increasingly greater quantities of posterior genes are expressed. For example in the group 4 triple mutant, the degree of competition against the more anterior genes would be low in C5, where only hoxc-4 (the fourth group 4 paralog) is strongly expressed and hoxa-5 and hoxb-5 are weakly expressed; greater in $\mathrm{C} 6$ and $\mathrm{C} 7$, where all of the group 5 genes are strongly expressed; and greatest in the thoracic vertebrae, where both group 5 and group 6 Hox genes are expressed. Correspondingly, C5 is strongly transformed, to a Cl identity; C6 and C7 are more weakly transformed, to a $\mathrm{C} 5$ and $\mathrm{C} 6$ identity; and the thoracic vertebrae are not transformed at all. Thus, the degree of anteriorization of these vertebrae inversely correlates with the quantity of more posterior genes expressed, which is consistent with a quantitative posterior prevalence model. In Drosophila, the phenotypic suppression model (González-Reyes et al. 1990) is the equivalent to the posterior prevalence model in mouse. Similarly, in this model, there is not an absolute dominance of the more posterior genes and some abnormalities can occur in regions more posterior to the anterior limit of expression of the Drosophila HOM-C genes (Manak and Scott 1994).

Alternatively, the transformations in these more posterior vertebrae could be explained by more indirect effects. For instance, there could be secondary interactions that maintain anteroposterior order in the cervical vertebrae, so that the nearly complete transformations of identity at $\mathrm{C} 2$ and $\mathrm{C} 3$ lead to a secondary anteriorization of the remaining cervical vertebrae. Finally, the observation that the group 4 Hox paralogs function cooperatively in specifying regional identity of the axial skeleton suggests that similar mechanisms might also be operating in other group 4 Hox-expressing tissues.

\section{Materials and methods}

Skeleton preparations

Neonates were sacrificed, skinned, eviscerated, and fixed in $95 \%$ ethanol. Their skeletons were subsequently prepared by alkaline digestion and stained with alizarin red $\mathrm{S}$ for ossified bone and alcian blue 8GX for cartilage (Kochhar 1973).

\section{Southern analysis}

Tails were biopsied for DNA genotyping by Southern blot analysis \{Ramírez-Solis et al. 1993; Horan et al. 1994, 1995).

hoxb-5 immunohistochemistry

E12.5 embryos were processed for hoxb- 5 whole-mount immunohistochemistry as described (Wall et al. 1992; Rancourt et al 1995).

\section{Acknowledgments}

We thank Evelyn Berry for excellent animal care and are grateful to Nancy Wall for the generous gift of the hoxb-5 antibody. This work was supported by National Institutes of Health grants HD31568 to R.R.B. and HD18122 and HD28374 to D.J.W. and grants from the Medical Research Council of Canada and the National Cancer Institute of Canada to M.S.F., the Sid W. Richardson Foundation to R.R.B., and the International Human Frontiers of Science Program to D.J.W. A.B. is an Associate Investigator with the Howard Hughes Medical Institute. M.S.F. is a Chercheur-Boursier of the Fonds de la Recherche en Santé du Québec.

The publication costs of this article were defrayed in part by payment of page charges. This article must therefore be hereby 
marked "advertisement" in accordance with 18 USC section 1734 solely to indicate this fact.

\section{References}

Condie, B.G. and M.R. Capecchi. 1993. Mice homozygous for a targeted disnuption of Hoxd-3 (Hox-4.1) exhibit anterior transformations of the first and second cervical vertebrae, the atlas and axis. Development 119: 579-595.

1994. Mice with targeted disruptions in the paralogous genes hox $\mathrm{a}-3$ and hoxd -3 reveal synergistic interactions. $\mathrm{Na}$ ture 370: 304-307.

Duboule, D. 1991. Patterning in the vertebrate limb. Curr. Opin. Genet. Dev. 1: 211-216.

Duboule, D. and P. Dollé. 1989. The structural and functional organization of the murine $H O X$ gene family resembles that of Drosophila homeotic genes. EMBO I. 8: 1497-1505.

Duboule, D. and G. Morata. 1994. Colinearity and functional hierarchy among genes of the homeotic complexes. Trends Genet. 10: 358-364.

Featherstone, M.S., A. Baron, S.J. Gaunt, M.G. Mattei, and D. Duboule. 1988. Hox-5.1 defines a homeobox-containing gene locus on mouse chromosome 2. Proc. Natl. Acad. Sci. 85: 4760-4764.

Garcia-Fernàndez, J. and P.W.H. Holland. 1994. Archetypal organization of the amphioxus Hox gene cluster. Nature 370: 563-566.

Gaunt, S.J., P.T. Sharpe, and D. Duboule. 1988. Spatially restricted domains of homeo-gene transcripts in mouse embryos: Relation to a segmented body plan. Development (Suppl.) 104: 169-179.

Gaunt, S.J., R. Krumlauf, and D. Duboule. 1989. Mouse homeogenes within a subfamily, $\operatorname{Hox}-1.4,-2.6$ and -5.1, display similar anteroposterior domains of expression in the embryo, but show stage- and tissue-dependent differences in their regulation. Development 107: 131-137.

Gaunt, S.J., P.L. Coletta, D. Pravtcheva, and P.T. Sharpe. 1990. Mouse Hox-3.4: Homeobox sequence and embryonic expression patterns compared with other members of the Hox gene network. Development 109: 329-339.

Geada, A.M.C., S.J. Gaunt, M. Azzawi, S.M. Shimeld, J. Pearce, and P.T. Sharpe. 1992. Sequence and embryonic expression of the murine Hox-3.5 gene. Development 116: 497-506.

González-Reyes, A., N. Urquia, W.J. Gehring, G. Struhl, and G. Morata. 1990. Are cross-regulatory interactions between homeotic genes functionally significant? Nature 344: 78-80.

Graham, A., N. Paplopulu, J. Lorimer, J.H. McVey, E.G.D. Tuddenham, and R. Krumlauf. 1988. Characterization of a murine homeo box gene, Hox-2.6, related to the Drosophila Deformed gene. Genes \& Dev. 2: 1424-1438.

Graham, A., N. Paplopulu, and R. Krumlauf. 1989. The murine and Drosophila homeobox gene complexes have common features of organization and expression. Cell 57: 367-378.

Horan, G.S.B., K. Wu, D.J. Wolgemuth, and R.R. Behringer. 1994. Homeotic transformation of cervical vertebrae in Hoxa-4 mutant mice. Proc. Natl. Acad. Sci. 91: 12644 12648.

Horan, G.S.B., E. Kovacs, R.R. Behringer, and M.S. Featherstone. 1995. Mutations in paralogous Hox genes result in overlapping homeotic transformations: Evidence for unique and redundant function. Dev. Biol. 169: 359-372.

Jeanotte, L., M. Lemieux, J. Charron, F. Poirier, and E.J. Robertson. 1993. Specification of axial identity in the mouse: Role of the Hoxa-5 (Hox1.3) gene. Genes Dev. 7: 2085-2096.

Kaufman, T.C., R. Lewis, and B. Wakimoto. 1980. Cytogenetic analysis of chromosome 3 in Drosophila melanogaster: The homeotic gene complex in polytene chromosomal interval 84A,B. Genetics 27: 309-362.

Kochhar, D.M. 1973. Limb development in mouse embryos. I. Analysis of teratogenic effects of retinoic acid. Teratology 7: 289-298.

Kostic, D. and M.R. Capecchi. 1994. Targeted disruptions of the murine Hoxa-4 and Hoxa-6 genes result in homeotic transformations of components of the vertebral column. Mech. Dev. 46: 231-247.

Krumlauf, R. 1994. Hox genes in vertebrate development. Cell 78: 191-201.

Lawrence, P.A. and G. Morata. 1994. Homeobox genes: Their function in Drosophila segmentation and pattern formation. Cell 78: 181-189.

Lewis, E.B. 1978. A gene complex controlling segmentation in Drosophila. Nature 276: 565-570.

Manak, J.R. and M.P. Scott. 1994. A class act: Conservation of homeodomain protein functions. Development (Suppl.): 6171 .

Ramírez-Solis, R., H. Zheng, J. Whiting, R. Krumlauf, and A. Bradley. 1993. Hoxb-4 (Hox-2.6) mutant mice show homeotic transformation of a cervical vertebra and defects in the closure of the sternal rudiments. Cell 73: 279-294.

Rancourt, D.E., T. Tsuzuki, and M.R. Capecchi. 1995. Genetic interaction between hoxb-5 and hoxb-6 is revealed by nonallelic noncomplementation. Genes \& Dev. 9: 108-122.

Rubin, M.R., L.E. Toth, M.D. Pate, P. D'Eustachio, and M.C. Nguyen-Huu. 1986. A mouse homeo box gene is expressed in spermatocytes and embryos. Science 233: 663-667.

Scott, M.P. 1993. A rational nomenclature for vertebrate homeobox (HOX) genes. Nucleic Acids Res. 21: 1687-1688.

Slack, J.M.W., P.W.H. Holland, and C.F. Graham. 1993. The zootype and the phylotypic stage. Nature 361: 490-492.

Wall, N.A., C.M. Jones, B.L.M. Hogan, and C.V.E. Wright. 1992. Expression and modification of hox-2.1 protein in mouse embryos. Mech. Dev. 37: 111-120.

Wolgemuth, D.J., E. Engelmyer, R.N. Duggel, E. Gizang-Ginsberg, G.L. Mutter, C. Ponzetto, C. Viviano, and Z. Zakeri. 1986. Isolation of a mouse cDNA coding for a developmentally regulated, testis-specific transcript containing homeobox homology. EMBO J. 5: 1229-1235. 


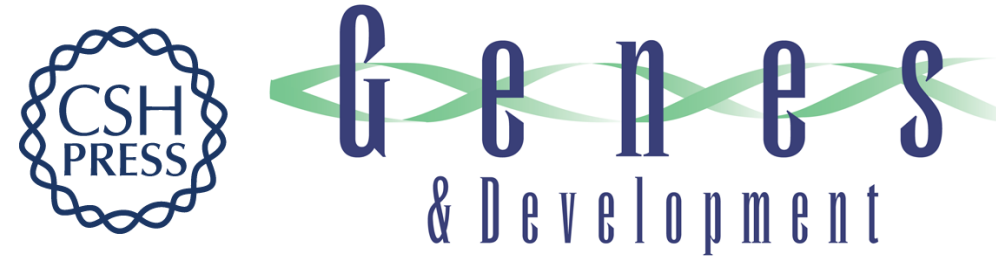

\section{Compound mutants for the paralogous hoxa-4, hoxb-4, and hoxd-4 genes show more complete homeotic transformations and a dose-dependent increase in the number of vertebrae transformed.}

G S Horan, R Ramírez-Solis, M S Featherstone, et al.

Genes Dev. 1995, 9:

Access the most recent version at doi:10.1101/gad.9.13.1667

References This article cites 30 articles, 11 of which can be accessed free at:

http://genesdev.cshlp.org/content/9/13/1667.full.html\#ref-list-1

License

Email Alerting

Service

Receive free email alerts when new articles cite this article - sign up in the box at the top right corner of the article or click here.

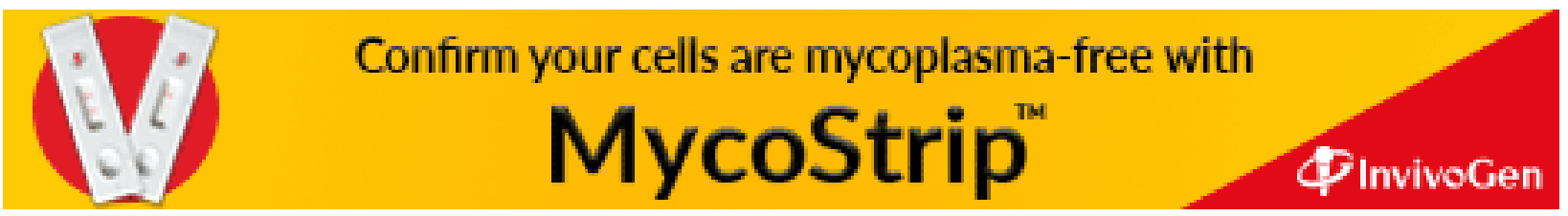

